\title{
MLSA phylogeny and antimicrobial susceptibility of clinical Nocardia isolates: a multicenter retrospective study in China
}

\author{
Ming Wei ${ }^{1}$, Xinmin Xu², Jingxian Yang ${ }^{3}$, Peng Wang ${ }^{1}$, Yongzhe Liư', Shuai Wang ${ }^{1}$, Chunxia Yang ${ }^{1}$ and Li Gu*
}

\begin{abstract}
Background: With the increase of detection rate and long treatment period, nocardiosis has become a noticeable problem in China. However, there are limited large-scale studies on the epidemiology and antimicrobial susceptibility profiles of clinical Nocardia spp. in China. The present study aimed to explore the species distribution and drug susceptibility pattern of 82 clinical Nocardia isolates from three tertiary hospitals in China by multilocus sequence analysis (MLSA) and broth microdilution (BMD) method.

Results: Pulmonary nocardiosis (90.2\%) was the most common clinical presentation of infection. N. cyriacigeorgica $(n=33 ; 40.2 \%)$ and $N$. farcinica $(n=20 ; 24.4 \%)$ were the most frequently encountered Nocardia species, followed by $N$. otitidiscaviarum ( $n=7 ; 8.5 \%), N$. abscessus $(n=5 ; 6.1 \%)$, N. asiatica $(n=4 ; 4.9 \%)$, and N. wallacei $(n=4 ; 4.9 \%)$. Trimethoprim/sulfamethoxazole (SXT) remained high activity against all Nocardia isolates (susceptibility rate: 98.8\%). Linezolid and amikacin were also highly active; 100 and $95.1 \%$ of all isolates demonstrated susceptibility, respectively. Except for N. otitidiscaviarum, all the Nocardia isolates exhibited high susceptibility rates to imipenem. The resistance rates of all isolates to clarithromycin and ciprofloxacin were 92.7 and $73.2 \%$, respectively, but the resistance rate of $\mathrm{N}$. farcinica to ciprofloxacin was only $25 \%$.

Conclusions: The clinically isolated Nocardia spp. had diverse antimicrobial susceptibility patterns, which were similar to the reports by other groups elsewhere, but some differences were also observed, mainly including imipenem and ciprofloxacin. According to this study, SXT still can be the first choice for empirical therapy due to the low resistance rate. Linezolid can be chosen when a patient is allergic to SXT, and amikacin and imipenem can be the choice in a combination regimen.
\end{abstract}

Keywords: Nocardia, MLSA, Antimicrobial susceptibility, Drug susceptibility pattern, China

\section{Background}

The genus Nocardia which is filamentous gram-positive bacterium and belongs to aerobic actinomycetes, exits in a wide range of environments [1-3]. More than 50

\footnotetext{
*Correspondence: guli2013227@foxmail.com

${ }^{1}$ Department of Infectious Diseases and Clinical Microbiology, Beijing Institute of Respiratory Medicine and Beijing Chao-Yang Hospital, Capital Medical University, 8 Gongren Tiyuchang Nanlu, Chaoyang District, Beijing 100020, People's Republic of China

Full list of author information is available at the end of the article
}

species can cause infections both in immunocompetent and immunocompromised individuals [4]. Due to the inexperience of the clinical laboratory technicians in primary hospitals in China, the particular characteristics of Nocardia growth, the unspecific pulmonary symptoms, as well as the low sensitivity of the culture-based method for diagnosis of nocardiosis [5-7], it is easy to miss detection of Nocardia in clinical practice. Partially, as a result, Nocardia infection is underestimated in China.

Nocardia species-level identification plays a crucial role in clinical therapy because particular species have 
specific drug susceptibility patterns $[8,9]$, but it is always a complex problem in clinical practice. With the development of matrix-assisted laser desorption ionizationtime of flight mass spectrometry (MALDI-TOF MS), the common clinical Nocardia species (e.g., N. cyriacigeorgica and $N$. farcinica) can be identified rapidly. However, the available database of MALDI-TOF MS is limited for Nocardia strains leading to some uncommon isolates with no or false identification [10,11]. The $16 \mathrm{~S}$ rRNA gene sequencing is generally considered as the primary means for accurate identification of the clinically encountered Nocardia isolates [12], but it cannot discriminate closely related species due to high conservation, unless it combines with a housekeeping gene, such as $\operatorname{gyr} B$ or $r p o B$ [13]. In recent years, multilocus sequence analysis (MLSA) has become more and more critical in bacterial taxonomy and species identification [14-16], because it has the advantages of a comparable database, robust discrimination, and relatively low cost. Our recent work shows that three-locus (gyrB-16S rRNA-secA1) MLSA for identification of clinical Nocardia species is superior to five-locus (gyrB-16S rRNA-secA1-hsp65-rpoB) MLSA which leads to misidentification for $N$. abscessus confirmed by digital DNA-DNA hybridization [17]. Therefore, the three-locus MLSA was carried out in this study to accurately identify clinical isolates from three tertiary care centers.

Although trimethoprim/sulfamethoxazole (SXT) has long been considered as the primary choice for therapy, it combines with other antibiotics that are always used for severe or systemic infections [12]. It is essential to carry out accurately antimicrobial susceptibility tests for clinical Nocardia isolates in China due to not only treatment for the individual patients but also providing guidance on empirical therapy in China.

The broth microdilution (BMD) method was recommended by the Clinical and Laboratory Standards Institute (CLSI) to determine antimicrobial susceptibility of Nocardia isolates [18], but few research groups carried out the susceptibility test with a relatively large number of isolates using this method in China $(<30$ strains in the related references) [19-21]. Therefore, this study aimed to provide the susceptibility profile of 82 clinical Nocardia isolates using the BMD method.

\section{Results}

\section{Species distribution and geographic characteristics}

In the phylogenetic tree formed by three-locus MLSA (Fig. 1), these sequence clusters were considered to represent species clusters [14]. A phylogenetic tree was constructed from the 1902-bp concatenated gyrB-16S rRNA-secA1 sequences of 23 Nocardia type strains and
82 clinical Nocardia isolates by the neighbor-joining method [22] and Kimura two-parameter distances [23].

Among the 82 isolates, 12 different species were represented. The two most common species were $N$. cyriacigeorgica $(n=33 ; 40.2 \%)$ and N. farcinica $(n=20$; $24.4 \%)$. Other species included $N$. otitidiscaviarum $(n=7 ; 8.5 \%), N$. abscessus $(n=5 ; 6.1 \%), N$. asiatica $(n=4$; $4.9 \%), N$. wallacei $(n=4 ; 4.9 \%), N$. puris $(n=2 ; 2.4 \%)$, $N$. aobensis $(n=1 ; 1.2 \%), N$. brasiliensis $(n=1 ; 1.2 \%), N$. nova $(n=1 ; 1.2 \%)$, novel species I $(n=3 ; 3.7 \%)$ and novel species II $(n=1 ; 1.2 \%)$ (Table 1$)$. The Novel species I and II were confirmed by digital DNA-DNA hybridization in our previous work because identification by five-locus MLSA and 16S rRNA gene phylogenetic tree had different results for these strains [17]. The majority of the Nocardia isolates were from the respiratory tract $(n=74$; $90.2 \%)$, followed by superficial abscess $(n=3 ; 3.7 \%)$, blood ( $n=2 ; 2.4 \%$ ), cerebrospinal fluid (CSF, $n=2 ; 2.4 \%$ ), and joint fluid $(n=1 ; 1.2 \%)$. N. cyriacigeorgica $(n=32$; 43.2\%) was the most frequently isolated Nocardia spp. from the respiratory tract. The isolates from blood, CSF and joint fluid were all $N$. farcinica $(n=5 ; 100 \%)$.

The geographic distribution of the clinical isolates is shown in Fig. 2. N. cyriacigeorgica and N. farcinica were the most widely distributed species in this study, being distributed in $64.3 \%(9 / 14)$ and $57.1 \%(8 / 14)$ of provinces, respectively. $N$. otitidiscaviarum isolates were distributed in eastern coastal regions (Beijing, Hebei, Jiangsu, and Zhejiang). N. abscessus isolates were distributed in three adjacent provinces of north China (Beijing, Hebei, and Shanxi).

\section{Demographic characteristics and infection types.}

The demographic characteristics and infection types of 82 patients with nocardiosis are shown in Table 2. More than half of the patients age ranged from 50 to 69 years old. Of the 82 patients, pulmonary infection was the primary type of infection.

\section{Antimicrobial susceptibility testing}

There are no CLSI clinical breakpoints for cefoxitin and tigecycline, but the studied 82 Nocardia clinical strains showed an overall susceptibility rate of the other 13 antimicrobial drugs according to species that is summarized in Table 1. In addition, the MIC range or value, $\mathrm{MIC}_{50}$ and $\mathrm{MIC}_{90}$ values for the 82 isolates are also summarized in Table 1 . The isolates demonstrated 98.8 and $100 \%$ susceptibility to SXT and linezolid, respectively. The susceptibility rates to amikacin, imipenem, tobramycin, ceftriaxone, minocycline, and amoxicillin/clavulanic acid were $95.1,73.2,63.4,42.7,42.7$, and $40.2 \%$, respectively, whilst the resistance rates to clarithromycin, ciprofloxacin, cefepime and moxifloxacin was 92.7, 73.2, 54.9, and 


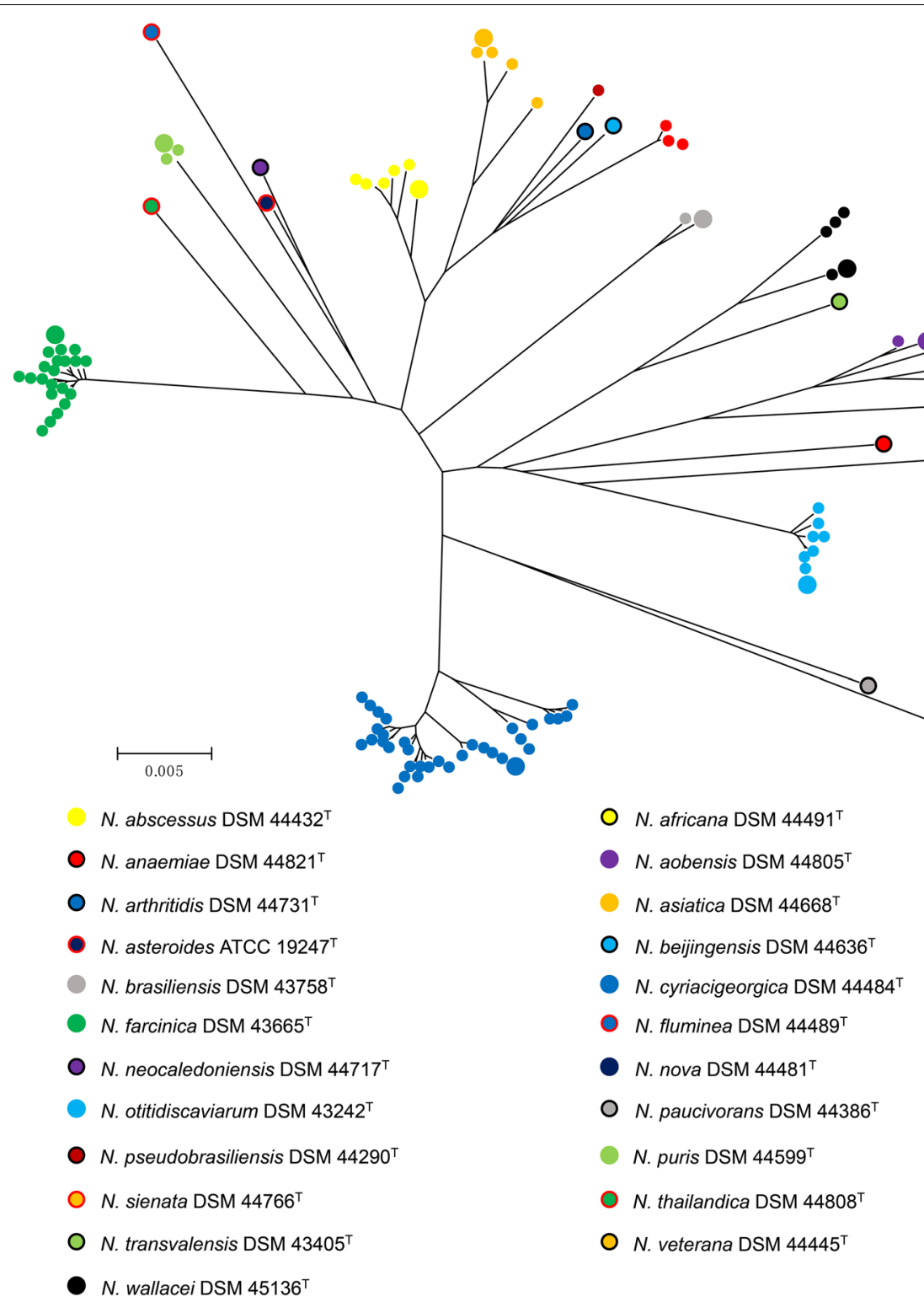

Fig. 1 Phylogenetic neighbor-joining tree was based on the MLSA analysis from the 1902-bp concatenated gyrB-16S rRNA-secA1 of the 23 Nocardia type strains and 82 Nocardia clinical strains. The isolates within each species cluster were assigned a color category according to their type strains. The big and small ball represented the type and clinical strains, respectively. The reliability of the topologies was assessed by the bootstrap method with 1000 replicates. ${ }^{\top}$, type strain

40.2\%. Although a low resistance level to doxycycline (9.8\%) was observed, a high percentage of isolates was in the intermediate category (65.9\%). The $\mathrm{MIC}_{50}$ and $\mathrm{MIC}_{90}$ for cefoxitin $(128$ and $>128 \mu \mathrm{g} / \mathrm{ml})$ of all Nocardia spp. were high, but for tigecycline $(2$ and $4 \mu \mathrm{g} / \mathrm{ml})$ were low, which is similar to the report by Tan et al. [24]

As previously described [12, 25], we noted a strong correlation between the drug susceptibility pattern types and Nocardia species. The type I, III, IV, V, and VI were displayed by the $N$. abscessus, $N$. nova, $N$. wallacei, $N$. farcinica, and N. cyriacigeorgica, respectively. In addition, the species that were not defined drug susceptibility pattern types also had unique drug susceptibility profiles. The susceptibility profiles were varied by species of Nocardia which are shown in Table S1. The susceptibility rates to amoxicillin/clavulanic acid and moxifloxacin were high for $N$. farcinica (90 and 75\%), but they were low for $N$. cyriacigeorgica (6.1 and 6.1\%), N. otitidiscaviarum (0 and 14.3\%), and N. asiatica (0 and 0), respectively. $N$. wallacei was the only species that was resistant 


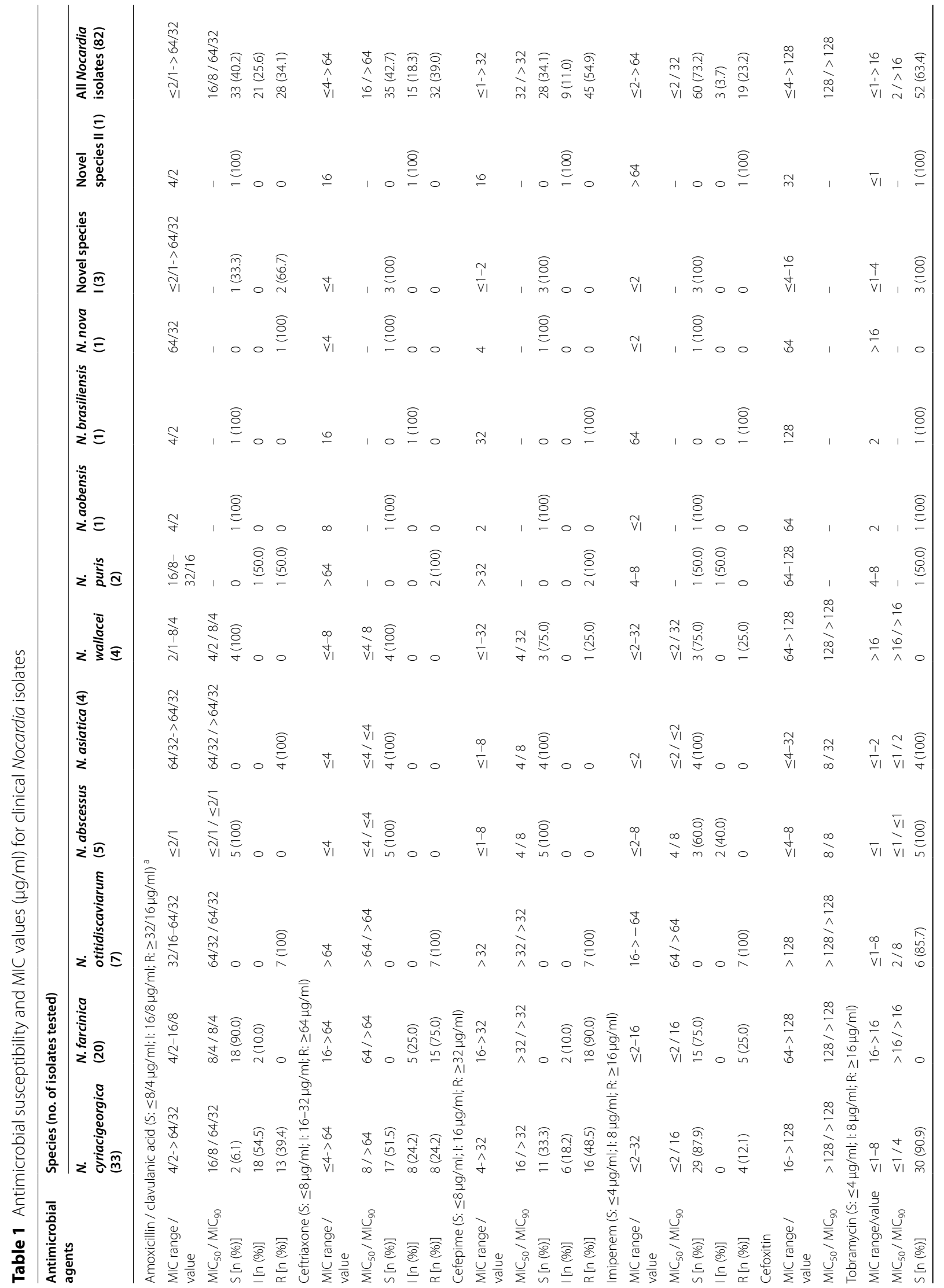




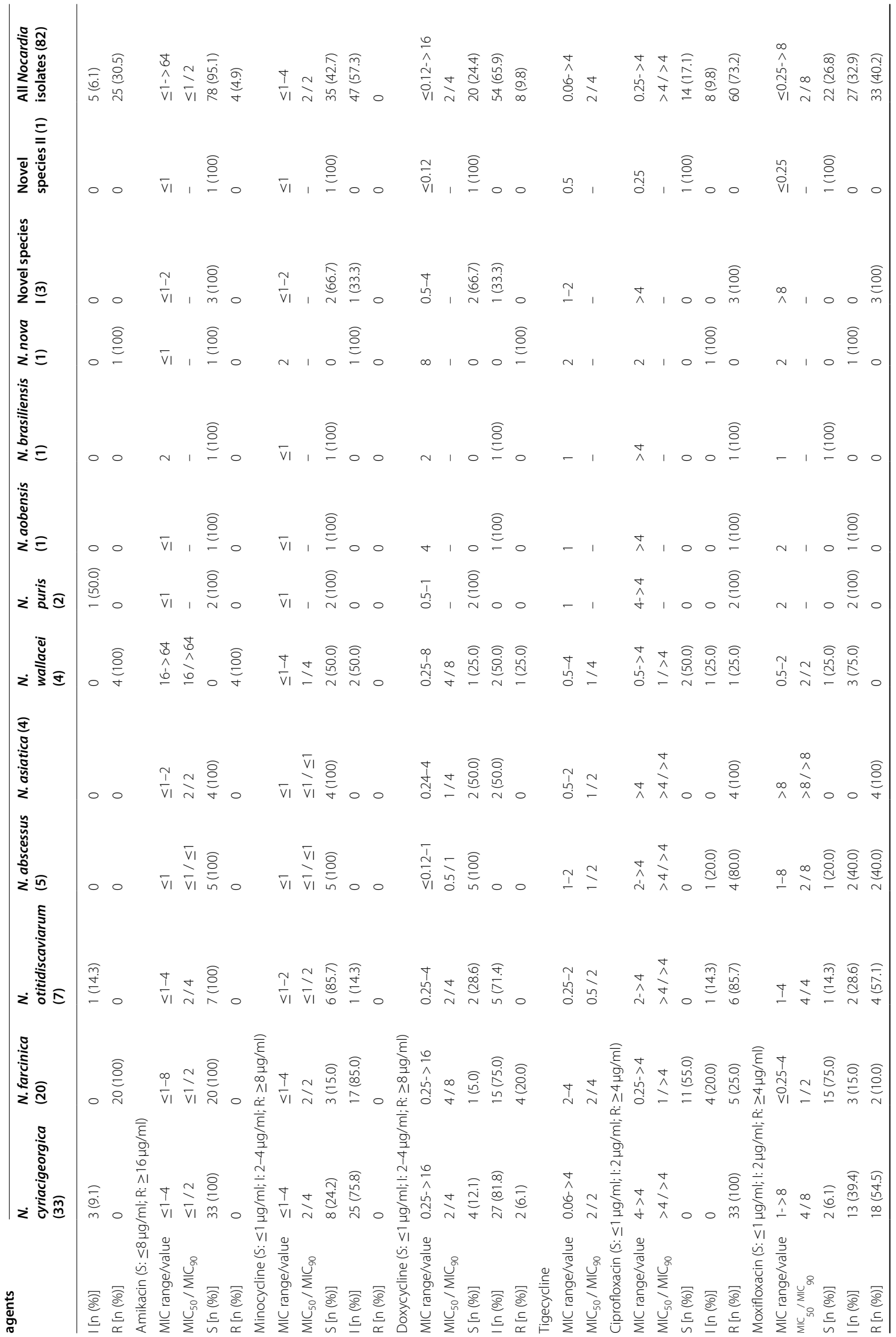




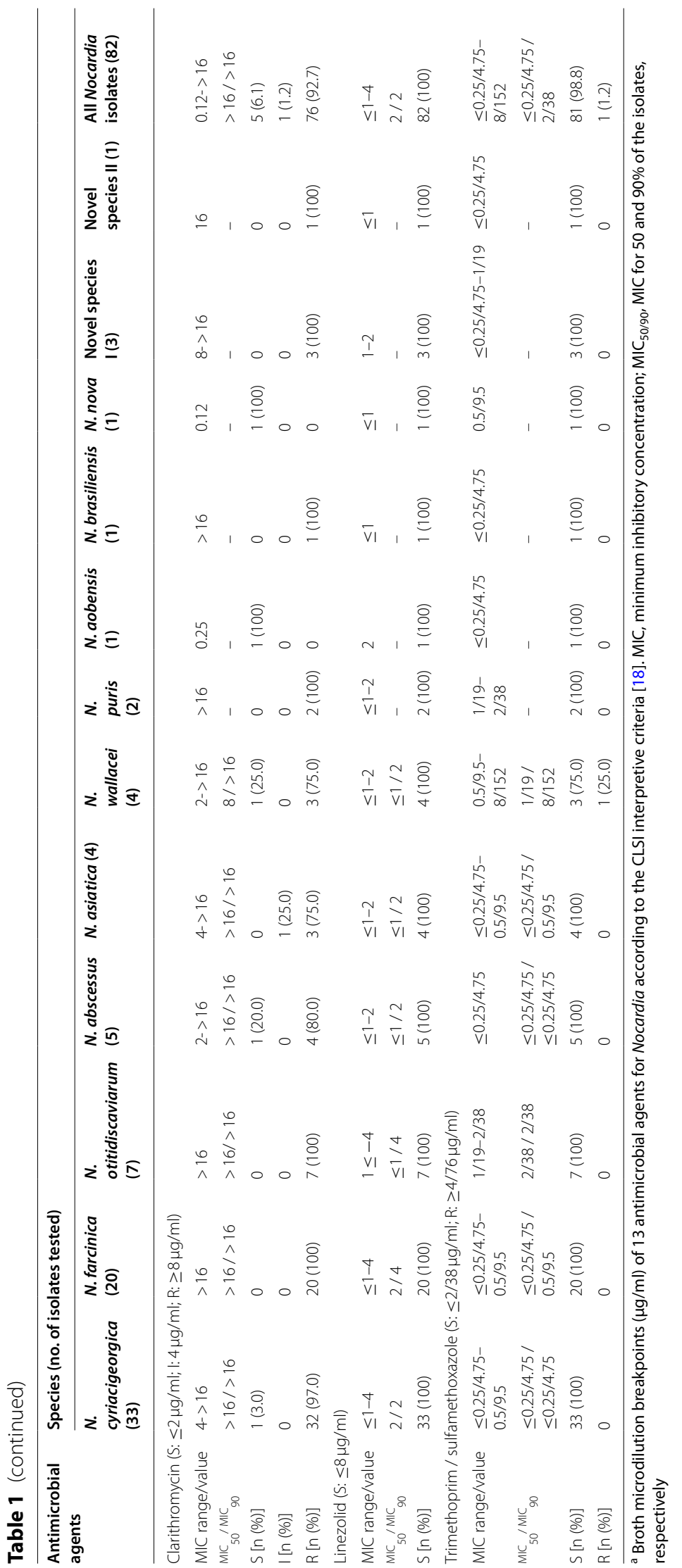




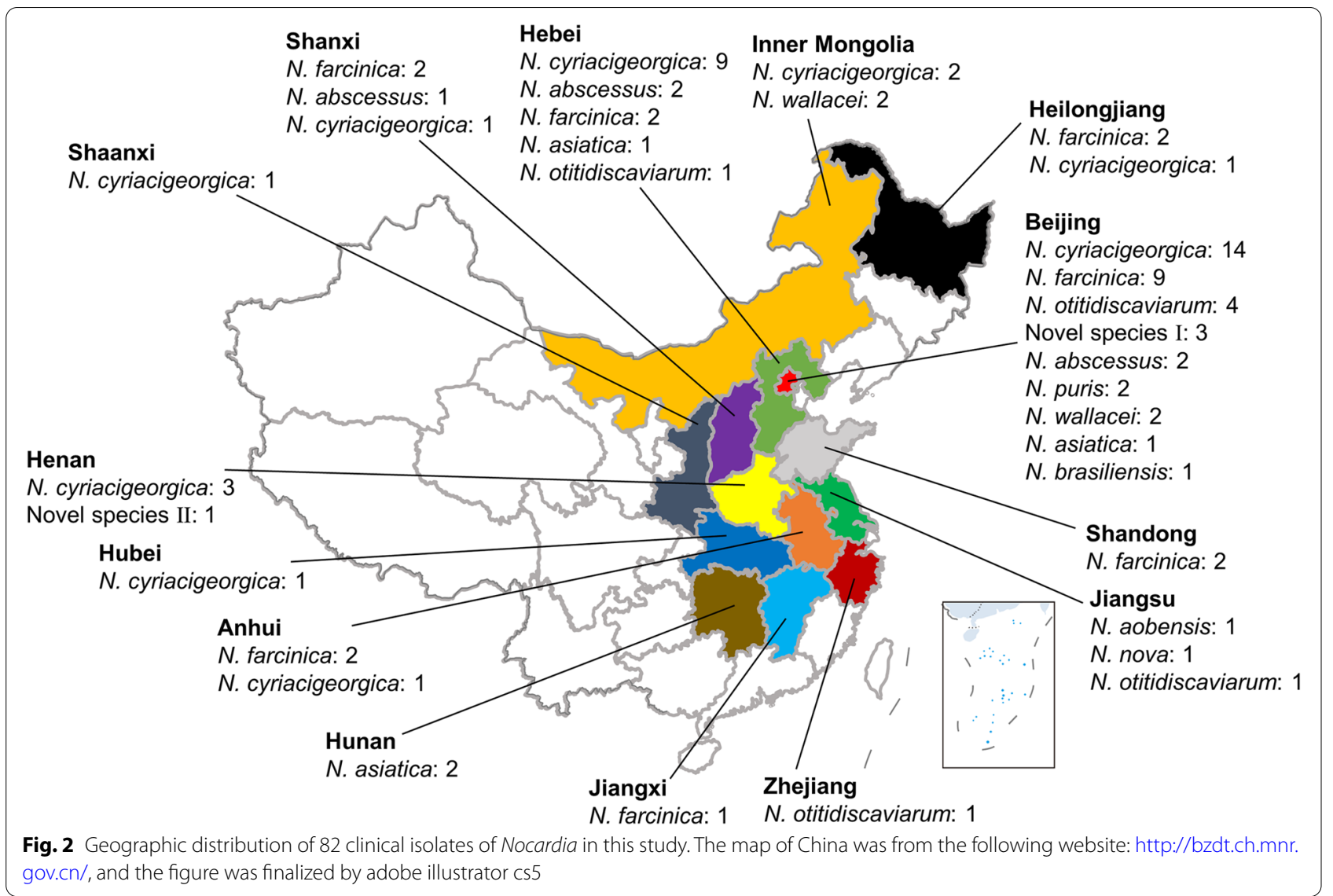

Table 2 Demographic characteristics and infection types of 82 patients with nocardiosis

\begin{tabular}{|c|c|c|}
\hline Characteristic & $\mathrm{n}^{\mathrm{a}}$ & $\%$ \\
\hline Age (Mean \pm S.D.) & $56.3 \pm 15.7$ & - \\
\hline $20-49$ & 22 & 26.8 \\
\hline $50-69$ & 47 & 57.3 \\
\hline $70-89$ & 13 & 15.9 \\
\hline \multicolumn{3}{|l|}{ Sex } \\
\hline Male & 49 & 59.8 \\
\hline Female & 33 & 40.2 \\
\hline \multicolumn{3}{|l|}{ Infection type } \\
\hline Disseminated infection ${ }^{b}$ & 5 & 6.1 \\
\hline Pulmonary infection & 74 & 90.2 \\
\hline Superficial infection & 3 & 3.7 \\
\hline
\end{tabular}

S.D Standard deviation. -, not applicable

${ }^{a}$ Data are $\mathrm{n}(\%)$ unless otherwise stated

${ }^{\mathrm{b}}$ The disseminated infection not involved the lung

to amikacin. Imipenem was effective against most Nocardia spp., but it was ineffective against $N$. otitidiscaviarum isolates.
Table S1 also shows that the drug susceptibility patterns reported by Wallace et al. [26] and Schlaberg et al. [25] The similarities and slight differences are all displayed.

\section{Discussion}

Due to the large number of Nocardia species, biochemical methods are insufficient to identify the clinically relevant species [4]. MALDI-TOF MS can rapidly identify the frequently encountered Nocardia species, but it is limited by the database and distinguishing closely related species [10]. The 16S rRNA gene sequencing is robust for accurate identification of Nocardia species, but it is also limited by discriminating closely related species due to high conservation [12]. The current study accurately identified the species by MLSA and determined the antimicrobial susceptibility by BMD method of clinically isolated Nocardia from three tertiary hospitals in China. The MLSA which is promising as the primary method in identification of prokaryotic species has powerful interspecies and intraspecies discrimination [14, 15, 27]. In our previous work, a fact has been proved by digital DNA-DNA hybridization analysis that three-locus MLSA is superior to five-locus MLSA for Nocardia species 
identification [17]. Therefore, three-locus MLSA was carried out in this study.

The geographical distribution of the Nocardia species has unique characteristics around the world. Figure 1 shows that $N$. cyriacigeorgica $(40.2 \%)$ was the most encountered species in this study that is similar to the reports from Iran, Spain, and the USA $[9,28,29]$. The prevalent species are slightly different in different regions [28, 30, 31]. Even within China, the prevalent species also have regional characteristics. $N$. otitidiscaviarum appears to be more prevalent in the eastern and southern coastal areas of China, and N. abscessus prefers to be distributed in the neighboring northern provinces of China, including Shanxi, Hebei, Beijing, and Shandong [20,32]. N. aobensis and N. nova which are close species on $16 \mathrm{~S}$ rRNA and/or secA1 genes and even classified into $N$. nova complex by Conville et al. [4], are distributed in Jiangsu in this study, while $N$. nova is distributed in Shandong in the study by Huang et al. [32] Actually, Jiangsu and Shandong are adjacent to each other. $N$. asiatica is mainly distributed in Hunan in this study, while it is distributed in Chongqing and Guangxi, which are adjacent to Hunan in the study by Huang et al. [32]

Based on the current study and the related references mentioned above [20,32], an interesting phenomenon was found: Some species prefer to be distributed according to the climate type, while others prefer to be distributed along the coast in China. Shanxi, Hebei, Beijing, and Shandong all belong to the monsoon climate of medium latitudes, and $N$. abscessus is prevalent. Hunan, Chongqing, and Guangxi all belong to the subtropical monsoon climate, and N. asiatica is prevalent. N. otitidiscaviarum and $N$. nova complex tend to be distributed in coastal provinces (Beijing, Hebei, Shandong, Jiangsu, Zhejiang, and Guangxi). This phenomenon suggests that the distribution of Nocardia is affected by the climate type and the sea.

With the increase of sample size, the incidence of nocardiosis in men increased significantly, which is different from our previous conclusion that there is no difference between men and women [19], but the new finding is similar to the report by Martínez-Barricarte, who summarized the gender distribution of patients with isolated nocardiosis worldwide [33]. According to Hernandez Hernandez et al., the female hormone estradiol shows inhibitory effect on Nocardia brasiliensis [34], which suggests the gender difference may be caused by estradiol. The proportion of nocardiosis for age ranging from 50 to 69 years was more than half in this study (58.5\%), which is similar to the report by Huang et al. (54.7\%) [32], but it is different from the data reported by Martínez-Barricarte that age ranging from 31 to 40 and from 51 to 60 years are the maximum proportion [33]. It suggests that nocardiosis in China has unique characteristics in terms of age. Nocardiosis most often shows up as a pulmonary infection $[1,12]$, confirmed by this study (90.2\%).

There are few large-scale studies [20, 21] about the antimicrobial susceptibility pattern of various Nocardia species based on BMD method that is recommended by the CLSI [18] in China. This study provides a relatively large collection of clinical Nocardia isolates to explore the correlation between antibiotics and species and reach a guideline for the nocardiosis treatment in China. SXT is the drug of the first choice for the treatment of nocardiosis, but some studies report a high level of resistance to the drug [35]. It is urgent to survey the SXT susceptibility in China. Overall, $98.8 \%$ of isolates were susceptible to SXT, and only one isolate of $N$. wallacei was resistant to the drug (Table 1). Similar results are reported by Lu et al. and other researchers [20,36-39]. Therefore, the present study indicates that SXT remains high-level of activity against Nocardia in vivo and can still be considered as the first-line therapeutic drug of choice for nocardiosis in China. The discrepancy may be caused by geographic differences, and/or SXT exposure before testing [36]. According to the reports [28, 31], the isolation rates of $N$. nova complex and $N$. farcinica which have high resistance rates to SXT were more than $10 \%$ in Spain and the USA. However, the former is rarely isolated in China [20, $32]$, and the latter may be divided into different types due to the different geographic areas.

Linezolid, that shows $100 \%$ activity against clinical Nocardia isolates in several large-scale studies [24, 25, $35,38,40]$, has become popular in treating nocardiosis recently, and the present study showed the same results. Amikacin is also an effective drug for all Nocardia isolates except for $N$. transvalensis complex, which is intrinsic resistant to the drug [37, 38, 40-42]. However, $N$. transvalensis complex is not prevalent in China [20,32]. In the study, $N$. wallacei, which belongs to $N$. transvalensis complex, is the only resistant species to amikacin. The treatment of nocardiosis commonly requires a combination of antibiotics. Imipenem is usually used in combination with SXT for adequate therapy of invasive Nocardia infections [43]. Imipenem showed good activity (susceptibility rate: $73.2 \%$ ) in the current study which supports it to participate in the combination regimen in empirical therapy. Amoxicillin/clavulanic acid, minocycline, or doxycycline can be also selected in a combination therapy, because the frequently encountered Nocardia species exhibit low-level resistance to the three antibiotics in China.

However, the remaining antimicrobials showed low activity against Nocardia isolates, and the susceptibility 
had species-specific. Compared with the studies by Wallace et al. [26] and Schlaberg et al. [25], a similar correlation between the antimicrobials and Nocardia species is observed, but there are some differences as well (Table S1). In particular, Wallace et al. reported that $N$. abscessus was resistant to imipenem, but the resistance rates were $40 \%$ in this study and $69 \%$ in the study by Schlaberg et al. Unlike Wallace et al. and Schlaberg et al., who indicated that $N$. wallacei was susceptible to ciprofloxacin, the current study indicated $50 \%$ susceptibility rate to the drug. For N. farcinica, Wallace et al. and this study reported that it was susceptible to imipenem, while Schlaberg et al. reported that the susceptibility rate was only $33 \%$. Besides, ciprofloxacin was less active to $N$. farcinica in the study by Schlaberg et al. and the current study, but was active in the study by Wallace et al. However, moxifloxacin, which is a higher generation quinolone antibiotic, was much more active than ciprofloxacin against $N$. farcinica in the study by Schlaberg et al. (susceptibility rate: $79 \%$ ) and the current study (75\%). It may be caused by the longer exposure time of ciprofloxacin compared to moxifloxacin [44]. For $N$. cyriacigeorgica, this study indicated that it was susceptible to imipenem (87.9\%), which is similar to the study by Wallace et al., but Schlaberg et al. reported the susceptibility rate was only $43 \%$. For $N$. otitidiscaviarum, ciprofloxacin was much active in the study by Wallace et al., but it was almost inactive in the study by Schlaberg et al. and the present study. The small differences mentioned above need to be further confirmed because the reproducibility of the BMD method for susceptibility testing of Nocardia species is not always very stable reported by Conville et al. [45]

For empirical treatment of nocardiosis, clarithromycin should be avoided due to the high resistance rate in this study. Ciprofloxacin is much less active to $N$. cyriacigeorgica, and ceftriaxone, cefepime, and tobramycin are much less active to $N$. farcinica. Therefore, these antibiotics also should avoid being used unless the species has been identified and/or the susceptibility test has been done, as $N$. cyriacigeorgica and $N$. farcinica are the most prevalent strains in China.

In addition to the major epidemic strains in China, the isolation rate of $N$. otitidiscaviarum in eastern and southern coastal areas is relatively high. The use of $\beta$-lactam antibiotics should be paid attention to because of its high resistance rate to this kind of antibiotics [20,32].

\section{Conclusion}

In summary, $N$. cyriacigeorgica and $N$. farcinica, which are widely distributed in China, were the most frequently isolated species, as well as they were the most common species causing pulmonary infection in this study. The clinically isolated Nocardia spp. had diverse antimicrobial susceptibility patterns, which were similar to the reports by other groups elsewhere, but some differences were also observed. It indicates the specific characteristics and provides the basis of empirical therapy in China. According to the current study, SXT can still be the first choice due to the low resistance rate. Linezolid can be chosen when a patient is allergic to SXT, and amikacin and imipenem can be the choice in a combination regimen.

\section{Methods \\ Strains}

A total of 82 non-repetitive clinical isolates of Nocardia from three tertiary hospitals in Beijing were studied between 2010 and 2020; seventy were from Beijing ChaoYang Hospital, of which 26 isolates have been tested for antimicrobial susceptibility with self-made drug susceptibility plates and published in our previous work [19], seven from Beijing Ditan Hospital, and five from Aerospace Center Hospital. The three hospitals have 1880, 1158 , and 1050 beds, respectively. Observation of grampositive beaded branching filaments on a direct gramstained smear and positive Kinyoun acid-fast stain under the microscope, as well as white to orange colonies on culture plates, indicated that the isolates were presumptive Nocardia [46]. The type strains in this study were selected as previously described [17]. In short, 23 type strains were used to construct phylogenetic relationships based on three-locus MLSA (gyrB-16S rRNA-secA1), and their GenBank accession numbers were shown in Table S2. Most of the type strains were selected based on their similarity to $16 \mathrm{~S}$ rRNA gene sequences of the clinical isolates.

\section{DNA extraction, $\mathrm{PCR}$, and sequencing}

The DNA was extracted by boiling method, which was the same as our previous work [17]. The primers of $16 \mathrm{~S}$ rRNA gene, and the primers of $g y r B$ gene and secA 1 gene referred to the work published by Carrasco et al. [13] and McTaggart et al. [16], respectively. The forward and reverse primers were listed in Table S3. The PCR experiments were carried out as previously described $[13,16]$. An ABI 3730XL DNA sequencer (Applied Biosystems) was used to sequence the PCR products, and the SeqMan program in Lasergene 7.1 (DNASTAR, Inc., Madison, WI) was used to assemble the sequences.

\section{Construction of phylogenetic tree}

The gene sequences of $\operatorname{gyr} B, 16 \mathrm{~S}$ rRNA, and $\sec A 1$ was aligned and trimmed by Mega (version 6.0) software [47] to generate the fragments of $482 \mathrm{bp}, 1026 \mathrm{bp}$, and $394 \mathrm{bp}$, respectively. The concatenation of gyrB-16S rRNA-secA1 (1902 bp sequence) was used to constructed phylogenetic 
tree by Mega software. The tree was computed by the neighbor-joining method [22] and Kimura two-parameter distances [23]. The bootstrap method with 1000 replicates was used to ensure the reliability of the topologies.

\section{Antimicrobial susceptibility testing by broth microdilution method}

Antimicrobial susceptibility testing of all isolates to 15 antimicrobial agents [amikacin $(1-64 \mu \mathrm{g} / \mathrm{ml})$, amoxicillin/clavulanic acid $(2 / 1-64 / 32 \mu \mathrm{g} / \mathrm{ml})$, cefepime $(1-32 \mu \mathrm{g} /$ $\mathrm{ml})$, cefoxitin $(4-128 \mu \mathrm{g} / \mathrm{ml})$, ceftriaxone $(4-64 \mu \mathrm{g} / \mathrm{ml})$, ciprofloxacin $(0.12-4 \mu \mathrm{g} / \mathrm{ml})$, clarithromycin $(0.06-16 \mu \mathrm{g} /$ $\mathrm{ml})$, doxycycline $(0.12-16 \mu \mathrm{g} / \mathrm{ml})$, imipenem $(2-64 \mu \mathrm{g} /$ $\mathrm{ml})$, linezolid $(1-32 \mu \mathrm{g} / \mathrm{ml})$, minocycline $(1-8 \mu \mathrm{g} / \mathrm{ml})$, moxifloxacin $(0.25-8 \mu \mathrm{g} / \mathrm{ml})$, tigecycline $(0.015-4 \mu \mathrm{g} / \mathrm{ml})$, tobramycin $(1-16 \mu \mathrm{g} / \mathrm{ml})$, and trimethoprim/sulfamethoxazole (SXT) $(0.25 / 4.75-8 / 152 \mu \mathrm{g} / \mathrm{ml})]$ was determined by BMD using the commercial Sensititre ${ }^{\mathrm{TM}}$ RAPMYCOI (Thermo Scientific, the United States). Briefly, $50 \mu \mathrm{l}$ of an organism suspension with a turbidity equivalent to $\sim 0.5$ McFarland standard was transferred to $10 \mathrm{ml}$ of Mueller Hinton II Broth (Becton, Dickinson and Company, the United States), and then $100 \mu$ l was inoculated into the microdilution wells to give a final concentration of $\sim 5 \times 10^{5} \mathrm{CFU} / \mathrm{ml}$ [18]. The microtitre plates were incubated aerobically at $35^{\circ} \mathrm{C}$ and were read after 3 days (or after 5 days if growth was insufficient after 3 days). Growth was examined daily by visual inspection. The minimum inhibitory concentration (MIC) was defined as the lowest concentration of drug that inhibited visible growth, except for SXT where the MIC was the $80 \%$ inhibition endpoint of growth compared with the control. Breakpoints for susceptibility and resistance were as defined by the CLSI (Table 1) [18]. Nocardia asteroides ATCC 19247, Staphylococcus aureus ATCC 29213 and Escherichia coli ATCC 35218 (for AMC only) were used as quality control strains.

\section{Supplementary Information}

The online version contains supplementary material available at https://doi. org/10.1186/s12866-021-02412-x.

Additional file 1: Table S1. Comparison of drug susceptibility patterns with clinical Nocardia species. Table S2. GenBank accession numbers of gene sequences for Nocardia type strains in the study. Table S3. The primer sequences used in this study.

\section{Acknowledgements}

The authors would like to thank all staff members of the Department of Infectious Diseases and Clinical Microbiology, Beijing Chao-Yang Hospital (Beijing, China) for their contribution to this work.

\section{Authors' contributions}

LG and MW conceived the study. MW, XX, JY, PW, YL, SW and CY performed the research. LG, MW, PW, XX, JY, and SW analyzed data and wrote the paper. All authors read and approved the manuscript.

\section{Funding}

This work was supported by the National Science and Technology Major Project (Grant number 2015ZX10003003). The funder played no role in the design of the study, nor in the collection, analysis, and interpretation of data, nor in writing the manuscript.

\section{Availability of data and materials}

The datasets generated and analysed during the current study are available in the Genome Sequence Archive (Genomics, Proteomics \& Bioinformatics 2021) in National Genomics Data Center (Nucleic Acids Res 2021), China National Center for Bioinformation / Beijing Institute of Genomics, Chinese Academy of Sciences (GSA: CRA005268) that are publicly accessible at: https://bigd.big.ac. cn/gsa/browse/CRA005268.

\section{Declarations}

\section{Ethics approval and consent to participate}

The current study was approved by the ethics committee of Beijing ChaoYang Hospital, Capital Medical University, Beijing, China. As this is a retrospective study and the benefits and privacy of patients in the study were not involved, the informed consents of patients were waived by the ethics committee of Beijing Chao-Yang Hospital, Capital Medical University. All experiments in the study were performed in accordance with relevant guidelines and regulations.

\section{Consent for publication}

Not applicable.

\section{Competing interests}

The authors declare that they have no competing interests.

\section{Author details}

${ }^{1}$ Department of Infectious Diseases and Clinical Microbiology, Beijing Institute of Respiratory Medicine and Beijing Chao-Yang Hospital, Capital Medical University, 8 Gongren Tiyuchang Nanlu, Chaoyang District, Beijing 100020, People's Republic of China. ${ }^{2}$ Department of Clinical Laboratory, Beijing Ditan Hospital, Capital Medical University, Beijing, People's Republic of China. ${ }^{3}$ Department of Clinical Laboratory, Aerospace Center Hospital, Beijing, People's Republic of China.

Received: 28 April 2021 Accepted: 6 December 2021

Published online: 13 December 2021

\section{References}

1. Wilson JW. Nocardiosis: updates and clinical overview. Mayo Clin Proc. 2012;87(4):403-7.

2. Kurup PV, Randhawa HS, Sandhu RS. A survey of Nocardia asteroides, $N$. caviae and N. brasiliensis occuring in soil in India. Sabouraudia. 1968;6(3):260-6.

3. Han HJ, Kwak MJ, Ha SM, Yang SJ, Kim JD, Cho KH, et al. Genomic characterization of Nocardia seriolae strains isolated from diseased fish. Microbiologyopen. 2019;8(3):e00656.

4. Conville PS, Brown-Elliott BA, Smith T, Zelazny AM. The complexities of Nocardia taxonomy and identification. J Clin Microbiol. 2018:56(1):e01419-7.

5. Weng SS, Zhang HY, Ai JW, Gao Y, Liu YY, Xu B, et al. Rapid detection of Nocardia by next-generation sequencing. Front Cell Infect Microbiol. 2020;10:13.

6. Rodriguez-Nava V, Durupt $\mathrm{S}$, Chyderiotis $\mathrm{S}$, Freydière AM, Karsenty J, de Montclos M, et al. A French multicentric study and review of pulmonary Nocardia spp. in cystic fibrosis patients. Med Microbiol Immunol. 2015;204(4):493-504

7. Haussaire D, Fournier PE, Djiguiba K, Moal V, Legris T, Purgus R, et al. Nocardiosis in the south of France over a 10-years period, 2004-2014. Int J Infect Dis. 2017;57:13-20.

8. Glupczynski Y, Berhin C, Janssens M, Wauters G. Determination of antimicrobial susceptibility patterns of Nocardia spp. from clinical specimens by Etest. Clin Microbiol Infect. 2006;12(9):905-12. 
9. Hashemi-Shahraki A, Heidarieh P, Bostanabad SZ, Hashemzadeh M, Feizabadi MM, Schraufnagel D, et al. Genetic diversity and antimicrobial susceptibility of Nocardia species among patients with nocardiosis. Sci Rep. 2015;5:17862.

10. Durand T, Vautrin F, Bergeron E, Girard V, Polsinelli S, Monnin V, et al. Assessment of VITEK ${ }^{\circledR}$ MS IVD database V3.0 for identification of Nocardia spp. using two culture media and comparing direct smear and protein extraction procedures. Eur J Clin Microbiol Infect Dis. 2020;39(3):559-67.

11. Toyokawa M, Ohana N, Ueda A, Imai M, Tanno D, Honda M, et al. Identification and antimicrobial susceptibility profiles of Nocardia species clinically isolated in Japan. Sci Rep. 2021;11(1):16742.

12. Brown-Elliott BA, Brown JM, Conville PS, Wallace RJ Jr. Clinical and laboratory features of the Nocardia spp. based on current molecular taxonomy. Clin Microbiol Rev. 2006;19(2):259-82.

13. Carrasco G, Valdezate S, Garrido N, Villalón P, Medina-Pascual MJ, SáezNieto JA. Identification, typing, and phylogenetic relationships of the main clinical Nocardia species in Spain according to their gyrB and rpoB genes. J Clin Microbiol. 2013;51(11):3602-8.

14. Gevers D, Cohan FM, Lawrence JG, Spratt BG, Coenye T, Feil EJ, et al. Opinion: re-evaluating prokaryotic species. Nat Rev Microbiol. 2005;3(9):733-9.

15. Shneyer VS. On the species-specificity of DNA: fifty years later. Biochemistry (Mosc). 2007;72(12):1377-84

16. McTaggart LR, Richardson SE, Witkowska M, Zhang SX. Phylogeny and identification of Nocardia species on the basis of multilocus sequence analysis. J Clin Microbiol. 2010;48(12):4525-33.

17. Wei M, Wang P, Yang C, Gu L. Molecular identification and phylogenetic relationships of clinical Nocardia isolates. Antonie Van Leeuwenhoek. 2019;112(12):1755-66.

18. Clinical and Laboratory Standards Institute (CLSI). Susceptibility testing of mycobacteria, nocardiae, and other aerobic actinomycetes; approved standard-second edition CLSI document M24-A2. Wayne: CLSI; 2011.

19. Wei M, Wang P, Qu J, Li R, Liu Y, Gu L, et al. Identification and antimicrobial susceptibility of clinical Nocardia species in a tertiary hospital in China. J Glob Antimicrob Resist. 2017;11:183-7.

20. Lu SH, Qian ZW, Mou PP, Xie L. Clinical Nocardia species: identification, clinical characteristics, and antimicrobial susceptibility in Shandong, China. Bosn J Basic Med Sci. 2020;20(4):531-8.

21. Yi M, Wang $L$, Xu W, Sheng $L$, Jiang $L$, Yang $F$, et al. Species distribution and antibiotic susceptibility of Nocardia isolates from Yantai, China. Infect Drug Resist. 2019;12:3653-61.

22. Gascuel O. BIONJ: an improved version of the NJ algorithm based on a simple model of sequence data. Mol Biol Evol. 1997;14(7):685-95.

23. Kimura M. A simple method for estimating evolutionary rates of base substitutions through comparative studies of nucleotide sequences. J Mol Evol. 1980;16(2):111-20.

24. Tan YE, Chen SC, Halliday CL. Antimicrobial susceptibility profiles and species distribution of medically relevant Nocardia species: results from a large tertiary laboratory in Australia. J Glob Antimicrob Resist. 2020;20:110-7.

25. Schlaberg R, Fisher MA, Hanson KE. Susceptibility profiles of Nocardia isolates based on current taxonomy. Antimicrob Agents Chemother. 2014;58(2):795-800.

26. Wallace RJ Jr, Steele LC, Sumter G, Smith JM. Antimicrobial susceptibility patterns of Nocardia asteroides. Antimicrob Agents Chemother. 1988;32(12):1776-9.

27. Carrasco G, de Dios CJ, Garrido N, Valdezate S, Cantón R, Sáez-Nieto JA. Shortcomings of the commercial MALDI-TOF MS database and use of MLSA as an arbiter in the identification of Nocardia species. Front Microbiol. 2016;7:542.

28. Valdezate S, Garrido N, Carrasco G, Medina-Pascual MJ, Villalón P, Navarro AM, et al. Epidemiology and susceptibility to antimicrobial agents of the main Nocardia species in Spain. J Antimicrob Chemother. 2017;72(3):754-61.

29. Schlaberg R, Huard RC, Della-Latta P. Nocardia cyriacigeorgica, an emerging pathogen in the United States. J Clin Microbiol. 2008;46(1):265-73.

30. Lebeaux D, Bergeron E, Berthet J, Djadi-Prat J, Mouniée D, Boiron P, et al. Antibiotic susceptibility testing and species identification of Nocardia isolates: a retrospective analysis of data from a French expert laboratory, 2010-2015. Clin Microbiol Infect. 2019;25(4):489-95.

31. Hamdi AM, Fida M, Deml SM, Abu Saleh OM, Wengenack NL. Retrospective analysis of antimicrobial susceptibility profiles of Nocardia species from a tertiary hospital and reference laboratory, 2011 to 2017. Antimicrob Agents Chemother. 2020;64(3):e01868-19.

32. Huang $L$, Chen $X, X u H$, Sun L, Li C, Guo W, et al. Clinical features, identification, antimicrobial resistance patterns of Nocardia species in China: 2009-2017. Diagn Microbiol Infect Dis. 2019;94(2):165-72.

33. Martínez-Barricarte R. Isolated Nocardiosis, an unrecognized primary immunodeficiency? Front Immunol. 2020;11:590239.

34. Hernandez-Hernandez F, Lopez-Martinez R, Mendez-Tovar LI, ManzanoGayosso P. Nocardia brasiliensis: in vitro and in vivo growth response to steroid sex hormones. Mycopathologia. 1995;132(2):79-85.

35. Uhde KB, Pathak S, McCullum I Jr, Jannat-Khah DP, Shadomy SV, Dykewicz CA, et al. Antimicrobial-resistant Nocardia isolates, United States, 19952004. Clin Infect Dis. 2010;51(12):1445-8.

36. Brown-Elliott BA, Biehle J, Conville PS, Cohen S, Saubolle M, Sussland D, et al. Sulfonamide resistance in isolates of Nocardia spp. from a US multicenter survey. J Clin Microbiol. 2012;50(3):670-2.

37. Cercenado E, Marín M, Sánchez-Martínez M, Cuevas O, Martínez-Alarcón J, Bouza E. In vitro activities of tigecycline and eight other antimicrobials against different Nocardia species identified by molecular methods. Antimicrob Agents Chemother. 2007:51(3):1102-4.

38. Lowman W, Aithma N. Antimicrobial susceptibility testing and profiling of Nocardia species and other aerobic actinomycetes from South Africa: comparative evaluation of broth microdilution versus the Etest. J Clin Microbiol. 2010;48(12):4534-40.

39. McTaggart LR, Doucet J, Witkowska M, Richardson SE. Antimicrobial susceptibility among clinical Nocardia species identified by multilocus sequence analysis. Antimicrob Agents Chemother. 2015;59(1):269-75.

40. Larruskain J, Idigoras P, Marimón JM, Pérez-Trallero E. Susceptibility of 186 Nocardia sp. isolates to 20 antimicrobial agents. Antimicrob Agents Chemother. 2011;55(6):2995-8.

41. Minero MV, Marín M, Cercenado E, Rabadán PM, Bouza E, Muñoz P. Nocardiosis at the turn of the century. Medicine (Baltimore). 2009;88(4):250-61.

42. Tremblay J, Thibert L, Alarie I, Valiquette L, Pépin J. Nocardiosis in Quebec, Canada, 1988-2008. Clin Microbiol Infect. 2011;17(5):690-6.

43. Gombert ME, Aulicino TM. Synergism of imipenem and amikacin in combination with other antibiotics against Nocardia asteroides. Antimicrob Agents Chemother. 1983;24(5):810-1.

44. Andriole VT. The quinolones: past, present, and future. Clin Infect Dis. 2005;41 Suppl 2:S113-9.

45. Conville PS, Brown-Elliott BA, Wallace RJ Jr, Witebsky FG, Koziol D, Hall GS, et al. Multisite reproducibility of the broth microdilution method for susceptibility testing of Nocardia species. J Clin Microbiol. 2012;50(4):1270-80

46. McNeil MM, Brown JM. The medically important aerobic actinomycetes: epidemiology and microbiology. Clin Microbiol Rev. 1994;7(3):357-417.

47. Tamura K, Stecher G, Peterson D, Filipski A, Kumar S. MEGA6: molecular evolutionary genetics analysis version 6.0. Mol Biol Evol. 2013;30(12):2725-9.

\section{Publisher's Note}

Springer Nature remains neutral with regard to jurisdictional claims in published maps and institutional affiliations.

Ready to submit your research? Choose BMC and benefit from:

- fast, convenient online submission

- thorough peer review by experienced researchers in your field

- rapid publication on acceptance

- support for research data, including large and complex data types

- gold Open Access which fosters wider collaboration and increased citations

- maximum visibility for your research: over 100M website views per year

At BMC, research is always in progress.

Learn more biomedcentral.com/submissions 